\title{
Study of Junction Carbon Paste Electrode / Indigo Carmine / Mercury (II): Application of the Law Cottrell
}

\author{
N'driSeiny Roger ${ }^{1,2}$, Coulibaly Mariame ${ }^{2}$, Yao N'guessan Alfred ${ }^{2}$, Bamba Drissa $^{2}$, Zoro Ellogne ${ }^{2}$ \\ ${ }^{1,2}$ Université Félix Houphouët Boigny de Cocody, Laboratoire de Physique de la matière condensée et technologie, 01 BP V 3, Abidjan, \\ Côte d'Ivoire
}

${ }^{2}$ Laboratoire Des Sciences Physiques Fondamentales et Appliquées de l'Ecole Normale Supérieure d'Abidjan, 08BP 10 Abidjan 08 Abidjan Côte d'Ivoire

\begin{abstract}
Electrochemical detection of mercury (II) by the carbon paste electrode modified by indigo carmine in the differential pulse voltammetry is obtained by a complexation reaction. Complexation time, $\mathrm{pH}$ and temperature parameters which influence the formation of the complex were investigated. The formed $\mathrm{Hg}$-IC complex is electro inactive. Its adsorption to the surface of the electrode causes a decreasing variation of the intensity of the anodic peak current versus time. Applying the Cottrell law of the faradaic and adsorption current has achieved the equivalent electrical circuit modelling all phenomena at the interface of the sensor.
\end{abstract}

Keywords: detection, mercury (II), modified electrode, indigo carmine, Cottrell law.

\section{Introduction}

Current African companies face in recent decades to pollution by heavy metals such as lead, cadmium, zinc, mercury etc. All these metals have a harmful not to overlook. Mercury which is the object of our study is addressing reproductive and nervous system [1]. To fight against this growing pollution Africa turns to electrochemical sensors. These sensors are often simple and compact devices consist of two parts: a transducer and a sensitive layer. The transducer transforms the signal (bio) chemical-induced physicochemical interactions of the sensitive layer in a readily usable electrical signal.

The sensitive layer is composed of conductive polymer adsorbed on the surface of the working electrode and has a complexing power.

In the design of sensors the study of electrical phenomena and transport at the interface is much obscured. Yet these phenomena as well as the nature of the electrode influence the performance and selectivity of the sensor.

The present study was to develop an electrochemical sensor mercury from the dye indigo carmine (IC) used in textile [2] and in cosmetics [3] on a carbon paste working electrode by differential pulse voltammetry.

The structure of the indigo carmine (IC) is shown in Figure $1[1,4]$. The working electrode is rendered sensitive to mercury (II) through a thin polymer layer deposited on the surface of the electrode by indigo carmine [4] electro polymerization [5]. The oxidation product of indigo carmine in alkaline medium, the carmine dehydroindigo (DHIC) formed has selective groups on its surface.<smiles>[O][S+]([O-])(O[Na])c1ccc2c(c1)C(=O)N/C2=C1/NNC1=O</smiles>

Figure 1: Structure of the indigo carmine (IC)

The purpose of this paper is the study of the sensor interface and modeling of phenomena at the interface by enforcement Cottrelllaw to faradic and capacitive currents. The enforcement of law Cottrell to provide an electric equivalent circuit [6] to translate the phenomena at the interface of the sensor.

\section{Experimental}

\subsection{Equipment}

Electrochemical experiments were performed with a potentiostat Palm sense (Palm BV) controlled by the pstrace software and computer controlled. The analytical method is differential pulse voltammetry. The carbon paste electrode was used as working electrode (W), the electrode $\mathrm{Ag} / \mathrm{AgCl} / \mathrm{KCl}$ sat is used as a reference electrode (R) and platinum as counter electrode (C).

\subsection{Reagents and materials}

All chemicals used were of analytical purity. The carbon paste is graphite powder $(\Phi=0,22 \mu \mathrm{m})$ from SIGMAALDRICH, indigo carmine Aldrich Company.

Paraffin oil is of DP-PHARMA; sodium hydroxide $(\mathrm{NaOH})$ of Sharlau and mercury by MERCK. The alkaline solution of indigo carmine $\mathrm{pH}=10$ was prepared from distilled water and sodium hydroxide at room temperature $25^{\circ} \mathrm{C}+/-1$. 


\section{International Journal of Science and Research (IJSR) \\ ISSN (Online): 2319-7064}

Index Copernicus Value (2013): 6.14 | Impact Factor (2014): 5.611

\subsection{Preparation of the carbon paste electrode}

The carbon paste working electrode is prepared from the black graphite powder mixed with a hydrophobic binder and inactive: paraffin oil. Paraffin oil is mixed in the proportion of $0.3 \mathrm{ml}$ per $1 \mathrm{~g}$ of graphite. The mixture is pounded in a mortar with a pestle to a homogeneous paste. The mixed dough is put into the body of the geometric electrode area $0.07 \mathrm{~cm}^{2}$. It is regenerated by a regular wipe clean and smooth on the paper.

\subsection{Procedure}

The carbon paste working electrode and the other electrodes are immersed in the aqueous alkaline solution of indigo carmine. The cell is subjected to the potential window between $E_{\text {initial }}=-0,1 \mathrm{~V}$ and $E_{\text {final }}=0.7 \mathrm{~V}$. Potential instinct, conditioning, deposit and balance are $0,100 \mathrm{~V}$ respectively; $0,65 \mathrm{~V} ;-1,1 \mathrm{~V}$ and $0.05 \mathrm{~V}$. The time parameters are: time pulsation $0,07 \mathrm{~s}$ and 10 minutes complexation time. The production time of the oxidation reaction is $23 \mathrm{~s}$. The scan rate is $30 \mathrm{mV} / \mathrm{s}$.

\section{5 Procedure for determining}

The cell fed once to measure the oxidation peak of the indigo carmine without the presence of mercury (II), the peak falls, then added the desired amount of an aqueous solution of mercury (II), after a conditioning time of 10 minutes it takes up the peak. In the end each conditioning time of 10 minutes the various peaks are identified.

\section{Results and Discussion}

\subsection{Some useful relationships}

The Faradaic current is generated by the oxidation reaction of indigo carmine in the differential pulse voltammetry. The peak current is a function of the concentration and influenced by the microelectrode nature of the working electrode. Its peak current that obeys of the Cottrelllaw [79], solution of the second Fick law whose expression is given by the relationship (R1)

$\mathrm{I}(\mathrm{t})=\mathrm{nFSC} \mathrm{sol}^{\mathrm{s}}\left(\frac{1}{\sqrt{\pi \mathrm{Dt}}}+\frac{1}{\mathrm{r}_{0}}\right)(\mathrm{R} \mathbf{1})$

Where $\mathbf{n}$ is the number of electrons generated by the reaction, $\mathbf{F}$ is the Faraday constant $(=96,480) ; \mathbf{S}$ : the active surface of the electrode; $\mathbf{D}$ : the diffusion coefficient; $\mathbf{C}^{\text {sol }}$ the concentration of the red species; $t$ is time $\operatorname{andr}_{0}$ the radius of the working electrode.

This allows current from the physical characteristics of the electrode and of the reaction time to obtain the diffusion coefficient of the redox species. The thickness $(\delta)$ of the diffusion layer is expressed in the equation (R.2) $[9,10]$.

$$
\delta=\sqrt{\pi . \text { D.t }}(\mathbf{R . 2})
$$

with $\mathbf{t}$ the reaction time in seconds and $\mathbf{D}$ the diffusion coefficient of the redox species is obtained by calculation from (R1).

The electric charge of the diffusion layer is calculated using equation (R.3) connecting the intensity of the reaction time [10].
$Q=i \times t(R .3)$

The charge transfer resistance is obtained from the relationship Butler-Vollmer (R.4) [11-13]

$\mathbf{R}_{\mathrm{tc}}=\frac{\mathrm{RT}}{\mathrm{nFI}_{0}}(\mathbf{R . 4})$

$\mathbf{R}$ ideal constant gas, $\mathbf{T}$ is the temperature in degrees Kelvin, $\mathbf{F}$ Faraday's constant and $\mathbf{I}_{\mathbf{0}}=\mathbf{I} / \mathbf{S}$ the current density. The charge transfer resistance is related to the rate constant by the proportionality relation (R.5)

$\mathbf{k}^{0}=\frac{\mathbf{I}_{0}}{\text { nFSC }_{0}}(\mathbf{R . 5})$

The evolution of the faradic current as a function of concentration variations (or activity) redox species is described by the Warburg impedance (R.6) [11-13]

$\mathbf{W}^{*}(\mathbf{w})=\frac{\mathbf{R}_{\mathbf{t}} \times \mathbf{y}}{\sqrt{\mathbf{j} \mathbf{w}}}(\mathbf{R . 6})$

With $\mathbf{y}=\frac{\mathbf{K}_{\mathrm{red}}}{\sqrt{\mathbf{D}_{\mathbf{~ o x}}}}+\frac{\mathbf{K}_{\mathbf{o x}}}{\sqrt{\mathbf{D}_{\text {red }}}}$

In our case the second component of $\mathbf{y}$ will not be used due to the oxidation reaction irreversibility.

The introduction of mercury (II) ions in the reaction medium leads to the birth of a capacitive current. The adsorption current is inverse of the law of diffusion of Cottrell. Its expression is given by the relationship R.7)

$\operatorname{Ic}(t)=\frac{E_{s}}{R_{s}} \exp \left(-\frac{1}{R_{s} C}\right)+\frac{n_{F S D_{i}} C^{s o l}}{r_{0}}(R .7)$

With Es: the pulse potential, the resistance Rs of the solution and $\mathbf{C}$ the differential capacitance in equation (R.7), the limit current is: $\mathbf{I}_{\mathbf{l i m}}=\left(\frac{\mathbf{n F S D}_{\mathbf{i}} \mathbf{C}^{\text {sol }}}{\mathbf{r}_{\mathbf{0}}}\right)$, it depends on the radius $r_{0}$ of electrode and analyte diffusion coefficient complexing.

In the case of complexing with the detection process $\mathbf{C}^{\text {sol }}$ concentration of the metal deposited on the surface of the electrode is given by the formula (R.8) [14]:

$C_{j}=\frac{i_{j} t_{\text {acc }}}{n \text { F A }}(R .8)$

with $\mathbf{t}_{\text {ace }}$ the accumulation time, the time from which the current limit is established,

$\mathbf{i}_{\mathbf{j}}$ : The current limit, $\mathrm{n}$ the number of electrons F Faraday's constant and A geometric area of the electrode. The double layer capacity embodying the polymer-metal interface is given by the equation (R.9) [15].

$2 \pi$. f.R.C $\mathrm{dl}_{\mathrm{dl}}=1$ (R.9)

Where $\mathbf{f}$ is the frequency, $\mathbf{R}$ resistance of the solution and $\mathbf{C}_{\mathbf{d l}}$ of the electrochemical double layer capacity. The capacitor charge created by the formation of the double layer is given by the relation (R10). 


\section{International Journal of Science and Research (IJSR) \\ ISSN (Online): 2319-7064 \\ Index Copernicus Value (2013): 6.14 | Impact Factor (2014): 5.611}

$\mathbf{Q}_{\mathrm{C}}=\mathbf{C} \times \mathbf{E}_{\mathrm{S}}(\mathbf{R} 10)$

Es with the pulse potential and $\mathrm{C}$ differential capacity.

\subsection{Faradaic Process}

This alkaline solution of indigo carmine device and threeelectrode system is subjected to a potential sweep through the potentiostat. The polarization of the electrode and the consumption faradaic process IC (ICDHIC $+2 \mathrm{e}^{-}$) which follows have the consequence that a material transport limit to diffusion. The current faradaic obeys the law of diffusion of Cottrell.

The oxidation peak of the alkaline solution of indigo carmine concentration of $10^{-3} \mathrm{~mol} / \mathrm{L}$ measured by differential pulse voltammetry is to $10,578 \mu \mathrm{A}$ and appears at Eappl $=0,35 \mathrm{~V}$ (vs $\mathrm{Ag} / \mathrm{AgCl} / \mathrm{KCl})$. This peak in the shape of a Gaussian is shown in Figure 2a.

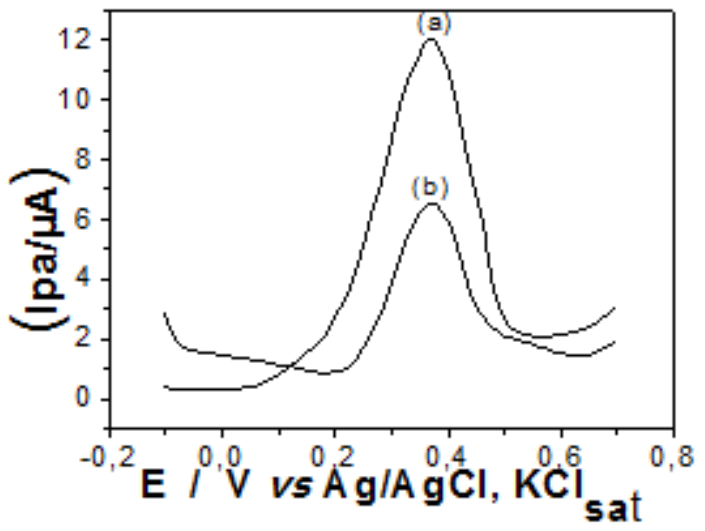

Figure 2: Differential pulse voltammogram at indigo carmine centration $\mathrm{C}=10^{-3} \mathrm{~mol} / \mathrm{L}$ at $\mathrm{pH}=10(\mathrm{a})$ : absence of mercury, (b): the presence of 100 ppb mercury concentration, pulse time $0,07 \mathrm{~s} ; \mathrm{E}=0,1 \mathrm{~V}$ pulsation, scanning speed $0,03 \mathrm{~V} / \mathrm{s}, 10 \mathrm{~min}$ reaction time

This oxidation reaction in alkaline medium (Figure 2.a) is irreversible [4] and is held at the spacing of the three phases shown in Figure 3 [16].

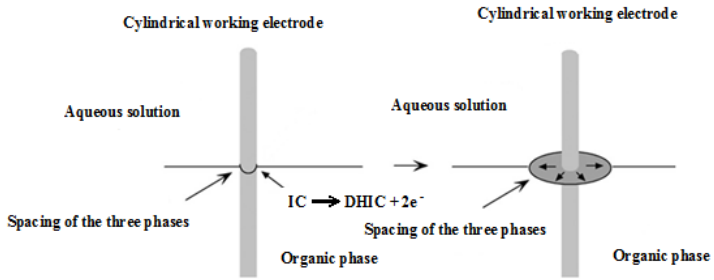

Figure 3: Diagram of the three-spaced solid / organic liquid / aqueous liquid

This phase junction Solid / organic / aqueous liquid fluid greatly influences the redox reaction, especially the organic phase (paraffin oil). This organic phase has the function of confining the reaction at the interface $[5,16]$. The characteristics of the faradaic reaction measured and calculated from expressions of faradic current are contained in Table 1.
Table 1 shows the comparison between the calculated values and those provided by the literature.

The analysis of the table provides information on the phenomena at the interface and on the kinetics of the reaction. The value of the diffusion coefficient $3, \mathbf{1 4 . 1 0}^{-6}$ $\mathrm{cm}^{2} \cdot \mathrm{s}^{-1}$ found on the base of the active area of the electrode $\left(\mathbf{A}=\mathbf{0}, \mathbf{0 7} \mathbf{~ c m}^{2}\right)$ is consistent with that reported in the literature for reactions in aqueous solution. According Maquere and al., the diffusion coefficient of the species electroactive in aqueous solution is between $\mathbf{1 0}^{-5}$ and $\mathbf{1 0}^{-6}$ [9].The constant value of kinetick $\mathbf{k}^{\mathbf{1}}=\mathbf{1}, \mathbf{7} \times \mathbf{1 0}^{-\mathbf{5}}$ $\mathbf{c m} / \mathbf{s s u g g e s t s}$ that electron transfer kinetics is slower.

Table 1: Characteristics of current Faraday on a geometric electrode area $\mathrm{A}=0.07 \mathrm{~cm}^{2}$

\begin{tabular}{|c|c|c|}
\hline Sizes & Values & $\begin{array}{l}\text { Literature } \\
\text { values }\end{array}$ \\
\hline $\begin{array}{l}\text { Diffusion coefficient of indigo } \\
\text { carmine }\left(\mathrm{cm}^{2} \cdot \mathrm{s}^{-1}\right)\end{array}$ & $3,14 \cdot 10^{-6}$ & $10^{-5}-10^{-6}[9]$ \\
\hline $\begin{array}{l}\text { Thickness of the diffusion layer } \\
(\mathrm{cm})\end{array}$ & 0,015 & \\
\hline electrical Charge & $2,4 \cdot 10^{-4} \mathrm{C}$ & $\begin{array}{c}2,5.10^{-4} \mathrm{C} \\
{[12]}\end{array}$ \\
\hline $\begin{array}{l}\text { The charge transfer resistance } \mathrm{R}_{\mathrm{tc}} \\
(\Omega)\end{array}$ & 1215 & $\begin{array}{c}6500-11000 \\
{[5]}\end{array}$ \\
\hline $\begin{array}{l}\text { Constant electronic transfer } \\
\text { kinetics } \mathrm{k}^{0}: \mathrm{cm} / \mathrm{s}\end{array}$ & $1,7 \times 10^{-5}$ & $10^{-7}[5]$ \\
\hline Warburg impedance $\left(\mathrm{j}^{-0,5} \Omega \mathrm{cm}^{2}\right)$ & 0,82 & $64,5[12]$ \\
\hline
\end{tabular}

This value also shows that the surface of the carbon paste electrode was activated by the indigo carmine adsorbed on its surface [5]. The low value of the $R_{t c}$ charge transfer resistance of a watch that electrons are not strongly related material.

The electrons which are involved in the oxidation reaction can easily be relocated.. It is therefore of $\pi$ electrons of the conjugated aromatic system [17].

On the other hand this value suggests that the sensitive layer formed on the surface of the electrode is not visible as the layers formed in the case of the modification of the electrode by proteins such as fibrinogen or transfer resistance of load is high [5].The low value of the Warburg impedance $\left(0.82 \Omega \mathbf{c m}^{2}\right)$ could be attributed to the decrease of the ion motion in the polymer electrolyte interface. The thickness of the diffusion layer $\mathbf{0 . 0 1 5} \mathrm{cm}$ is calculated ten times smaller than the radius $r_{0}$ of the cylindrical electrode $(0.15 \mathrm{~cm})$.

This result shows that the effects of edges of the electrode on the diffusion process are negligible and the diffusion regime is plan $[9,10]$.

\subsection{Capacitive Process}

\subsubsection{Mercury detection}

Detecting the complexation with indigo carmine mercury adsorbed to the surface of carbon paste electrode is made of differential pulse voltammetry. The addition of the 100 ppb mercury concentration after an incubation time of 10 minutes leads to the decrease in the anodic peak current (Figure 2.b). This decrease in peak current is due to the 


\section{International Journal of Science and Research (IJSR) \\ ISSN (Online): 2319-7064 \\ Index Copernicus Value (2013): 6.14 | Impact Factor (2014): 5.611}

formation of Hg-IC complex. This électroinactif complex is adsorbed on the surface of carbon paste electrode by specific reaction. The current decreases with time analysis.

\subsubsection{Influence of reaction time}

The evolution of the current vs. time has been studied and shown in Figure 4.

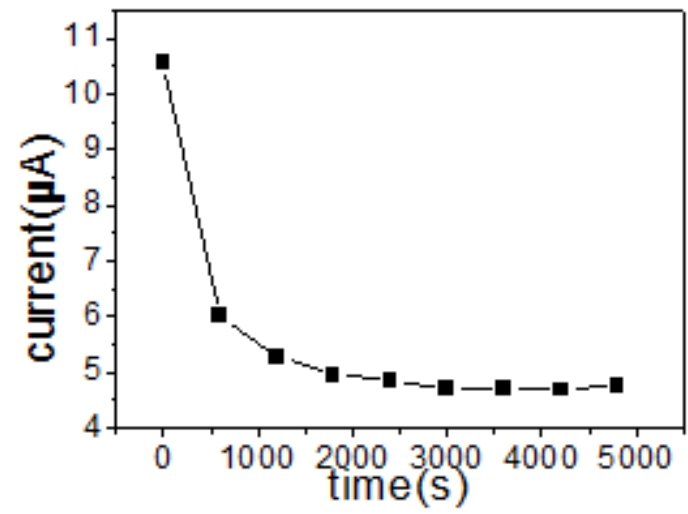

Figure 4: Evolution of the current versus time of 10${ }^{3} \mathrm{~mol} / \mathrm{L}$ indigo carmine solution at $\mathrm{pH}=10$ following the addition of the $100 \mathrm{ppb}$ mercury concentration, conditioning time $10 \mathrm{~min}$

This curve shows a nonlinear relationship between the evolution of the current and time. The current decreases after the addition of mercury.

He lowered over time to a limit value reached from 2000s.

This current is the sum of two terms, a time-dependent whose expression is identical to that of the adsorption current for a planar electrode and a constant term [10]. The constant term is the contribution related to the radial diffusion of mercury. Analysis of the curve as a function of time gives two information:

- In short times between 0 and 2000s the thickness of the adsorption layer is smaller than the radius of the electrode. The electrode acts as an electrode plate. Mass transport is dominated by a linear diffusion to the electrode surface. This is the time-dependent term in equation (R.7) that is predominant and current induced by application of the potential decreases with time according to the law of Cottrell.

- For longer time (time exceeds 2000s), the contribution of the first term decreases and becomes negligible. The mass transport process is then dominated by the radial diffusion. The current reaches steady state and is equal to the term independent of time

$\mathbf{I}_{\lim }==\frac{\mathrm{nFSD}_{\mathbf{i}} \mathrm{C}^{\mathrm{sol}}}{\mathbf{r}_{\mathbf{0}}}$

All these transport phenomena cause the structuration of the layers at the interface of the sensor shown in Figure 5.

Figure 5 is divided into two parts: Portions of the electrode side and a portion on the side of the electrolyte. The part of the side of the electrolyte layer comprises the Helmholtz, the diffuse layer of Gouy-Chapman and the homogeneous zone.

The thickness of the diffuse layer depends on the concentration and all forces applied to the loads. It is of the order of tens of Angstroms. There is significant potential drop across the Helmholtz layer.

It then rapidly attenuates in the Gouy-Chapman layer (Layer diffuse) to become homogeneous,

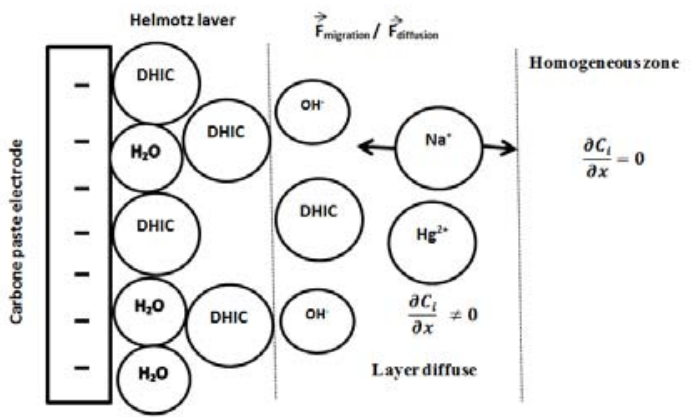

Figure 5: Structuring the sensor layers

when the electrolyte concentration becomes identical to that of the entire solution. Expression of the current equation (R.7) was modelled by a mathematical function whose expression is:

$$
i_{c}(t)=5,861 \cdot 10^{-6} e^{-0,002 . t}+4,717.10^{-6} A
$$

This feature justifies the observations described above. We distinguish a time related to component and a component independent of the time. The first part of the mathematical function describes the adsorption current on the electrode plate and the second current limit part obtained by radial spherical diffusion. The value of the current limit isi $\mathbf{i}_{\text {lim }}$ =4,717. $10^{-6}$ A. The curve in Figure 6 shows the relationship between the experimental curve and the theoretical curve obtained.

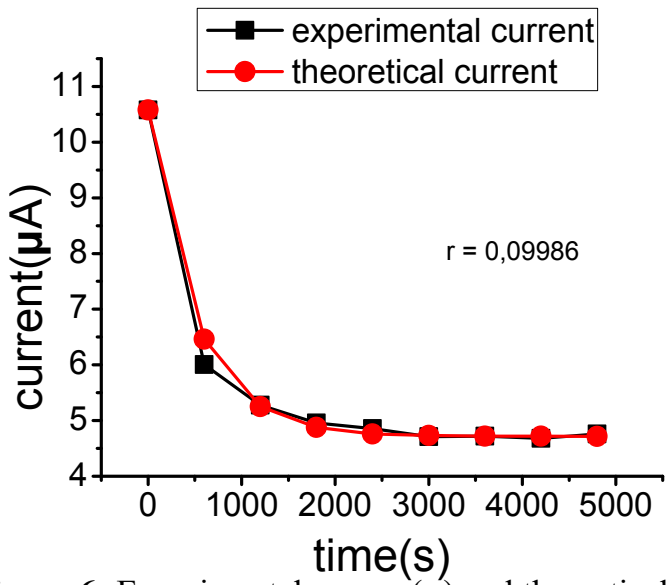

Figure 6: Experimental curves $(\mathbf{\bullet})$ and theoretical $(\bullet)$ of the current versus time

Figure 6 shows a very good agreement between the theoretical curve and the experimental curve with a correlation coefficient is $\mathrm{r}=0.9986$. 


\section{International Journal of Science and Research (IJSR) \\ ISSN (Online): 2319-7064 \\ Index Copernicus Value (2013): 6.14 | Impact Factor (2014): 5.611}

This theoretical curve was used to calculate certain characteristics of the solution. These characteristics compared to literature values are shown in Table 2.

The analysis of the table data showing a large value of the differential capacity and charging of the capacitor where the literature provides capacitance values of the order of $\mathrm{uF}$ [5]. These high values are related to the specificity of the reactions occurring at the electrode surface. According Jovic and al. [18], this differential capacitance is the sum of the capacitance of the double layer and the adsorption capacity.

Table 2: Characteristics of capacitive current $\mathrm{Hg}$-IC on a complex geometric electrode area $\left(\mathrm{A}=0,07 \mathrm{~cm}^{2}\right)$

\begin{tabular}{|l|c|c|}
\hline \multicolumn{1}{|c|}{ Characteristics } & Values & $\begin{array}{c}\text { Literature } \\
\text { values }\end{array}$ \\
\hline $\begin{array}{l}\text { Differential capacitance } \\
(\mu \mathrm{F})\end{array}$ & 29310 & $200[18]$ \\
\hline $\begin{array}{l}\text { capacitance of the double } \\
\text { layer }(\mu \mathrm{F})\end{array}$ & 18,7 & $0,3[5]$ \\
\hline The solution resistance $(\Omega)$ & 17062 & $320[5]$ \\
\hline The time constant $\left(\frac{1}{\tau}\right)(\mathrm{s})$ & 0,002 & $10^{-5}-10^{-6}[7]$ \\
\hline $\begin{array}{l}\text { Diffusion coefficient of } \\
\text { mercury ion }\left(\mathrm{cm}^{2} . \mathrm{s}^{-1}\right)\end{array}$ & $5.10^{-6}$ & $106[18]$ \\
\hline $\begin{array}{l}\text { The capacitor charge } \\
\left(\mu \mathrm{C} / \mathrm{cm}^{2}\right)\end{array}$ & 41900 & \\
\hline
\end{tabular}

The ability of the double layer is a function of frequency while the adsorption capacity is not.

The found value of mercury of the diffusion coefficient is consistent with the literature [9] and the radial diffusion phenomenon [10]

The resistance of the solution obtained in our study is large compared to that reported in the literature [5].

This large value of the resistance is related to the aqueous medium. Indeed according Migano and al, the aqueous medium is a resistive medium [19].The resistance of the solution and the time constant $\tau$ is influenced by the radius of the electrode. They are responsible for the phenomenon of ohmic drop $[19,20]$. The time constant $\boldsymbol{\tau}$ plays the role of filter that limits the variation of the electrode potential. More time constant $\tau$ is smaller (in the order of a millisecond) and the performance of the electrode are important.

\subsection{Modelling by an equivalent electrical circuit}

According to the literature the electrical circuit pattern in the absence of electroactive species is an RC series circuit $[14,21-23]$.

If an electroactive species in solution, before the introduction of the inactive species must take into account the faradaic impedance related to this faradic current. The total current appears as the sum of the capacitive current and the Faraday current. When the analysis time is long, the capacitive current in the presence of the inactive species becomes greater than the faradic current. It is represented by the differential capacitance in parallel with the faradaic impedance. The proposed model of the equivalent circuit without considering the specific surface of the carbon paste electrode reactions is shown in Figure 7.

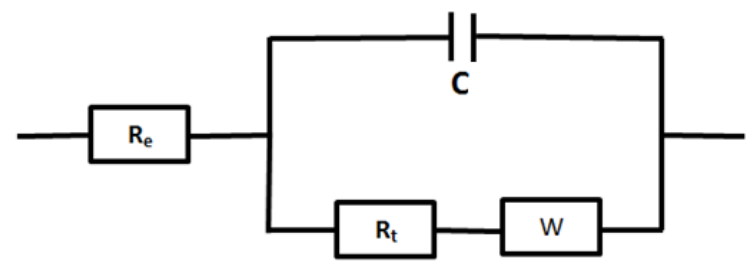

Figure 7: Equivalent circuit diagram of the mercury in electrochemical sensor

Re in this diagram refers to the resistance of the electrolyte or solution, the differential capacitance $\mathrm{C}\left(\mathrm{C}=\mathrm{C}_{\mathrm{dl}}+\mathrm{C}_{\mathrm{ads}}\right)$ and $\mathrm{W}$ the Warburg impedance.

\section{Conclusion}

A sensor associated with electrochemical techniques and the specific recognition process for biological agents immobilized on the working electrode. Analysis of the electrochemical interface of the sensor can include electrical process, transport (and mass distribution) and charge transfer at the interface solid electrode / electrolyte layers and structuring. The enforcement Cottrell realized at the interface of mercury sensor to determine the resistance of the reaction, the ability of the double layer and the diffusion coefficient and to establish the equivalent circuit. By intervening on these variables are optimized sensors.

\section{References}

[1] H.Tavallali, Spectrochimica Acta Part A 89 (2012) 216-221 :

[2] Colour Index, the Society of Dyers and Colourists. Revised third edition, UK, (1975).

[3] J.J. Berzas, J.R. Flores, M.J.V. Llerena, N.R. Farinas, Anal. Chim.Acta, 1999, 391, 353.

[4] M.Coulibaly, L.M. Muresan, STUDIA UBB CHEMIA, LVII, 3, 2012 (p. 65 - 72)

[5] Mama El Rhazi, PhD Thesis at the University Mohamed IV of Maroc, 1996

[6] Randles J. E. Trans. Farad. Soc., -44, 327, (1948).

[7] Amatore, C.; Pebay, C.; Thouin, L.; Wang, A.; Warkocz, J.-S. Analytical Chemistry 2010, 82, 69336939.

[8] Dekker, M. Physical Electrochemistry Principles, Methods, And Applications, Israel Rubinstein ed., 1995.

[9] F.Maquere, C. Frappart, Chronoamperometry and diffusion transportation, Bulletin of the Union of physicist's flight. 96 - February 2002

[10]C. Christopher, PhDThesis of the University of Toulouse (France), 2010

[11] François Henn Course electrochemistry-L3 M1 Chemical Physics and Materials contests, January 2006, France

[12] H.Imen, Ph.D. thesis INSA Lyon (France), 2009 


\section{International Journal of Science and Research (IJSR) \\ ISSN (Online): 2319-7064}

Index Copernicus Value (2013): 6.14 | Impact Factor (2014): 5.611

[13] Gerschel: Intermolecular Bindings Publisher: Intereditions / CNRS, 1995, chapter 3, 78-79.

[14] L. Baldriananova, I. Svançara, A. Economou. And S Sotiropoulos. Analyte chimica Acta.vol.580, $\mathrm{n}^{\circ} ; 1$. pp. $21-31,2006$

[15] Cole, K.S. Permeability and impermeability of cell membranes for ions. Cold Spring Harbor Symp. Quant. Biol. 1940, 8, 110-122.

[16]F. Scholz, S. Komorsky-Lovric', M. Lovric', Electrochem. Commun.2 (2000) 112.

[17]B. Mathieu, PhDThesis from the University of Lyon (France), 2011

[18] V.D. Jovic, Journal of Electroanalytical Chemistry 541 (2003) 1 / 11

[19] L.Mignano, $\overline{\mathrm{PhD}}$ Thesis, University of Nancy 1 (France), 1995

[20]A.Meunier, PhDThesis of the university Pierre and Marie Curie (France), 2011

[21]Fricke, H. Theory of electrolytic polarization. Philos. Mag. 1932, 14, 310-318.

[22] Daniel, V.V. Dielectric Relaxation; Academic Press: New York, 1967.

[23] Cole, K.S.; Cole, R.H. Dispersion and adsorption in dielectrics. I. Alternating current characteristics. J. Chem. Phys. 1941, 9, 341-351 